\title{
AN INTERPRETATION OF THE AMBIGUITY BETWEEN ANNUAL TERMS OBTAINED BY TIME AND LATITUDE OBSERVATIONS
}

\author{
TOYOZO OKUDA
}

International Latitude Observatory, Mizusawa, Japan

\begin{abstract}
Guinot and Feissel (1968), Okazaki and Nasaka (1970) reported the marked discrepancy between annual terms of the polar motion obtained by time and latitude observations. The disturbing effects of local non-polar terms in latitude variation on pole coordinates are discussed in the first half of this paper.

From an analogy between time and latitude observations, we can conclude that $\Delta x$ and $\Delta y$ in the pole coordinates obtained from time observations are proportional to a part of the common fictitious term $\Delta T$ in the rotation of the Earth. Using $\Delta T$ obtained from Okazaki's data for the same interval as Guinot's and Feissel's data, a test example is given as follows:

$$
\Delta T=5^{\mathrm{ms}} .1 \cos \left(2 \pi t+183^{\circ}\right), \quad \Delta x=0.52 \Delta T, \Delta y=-0.29 \Delta T .
$$

If we apply the above corrections to the pole coordinates obtained from time observations, we can derive the coordinates $x$ and $y$ which are comparable to those of the ILS. $\Delta \alpha_{\alpha}, \Delta S$ and local non-polar terms $\left(z_{5}-z_{4}\right)$ in the ILS latitude determination may be considered as the sources of $\Delta T$.
\end{abstract}

\section{In the Case of Latitude Variation}

We use the following formule for computing the polar coordinates $x, y$ and the common term $z$ independent of the polar motion.

$$
\mathrm{d} \varphi_{i}=x \cos \lambda_{i}+y \sin \lambda_{i}+z \quad(i \geqq 3) .
$$

The solutions of these formulae by the method of least squares are

$$
\begin{aligned}
& x=\sum a_{i} \mathrm{~d} \varphi_{i} \\
& y=\sum b_{i} \mathrm{~d} \varphi_{i} \\
& z=\sum c_{i} \mathrm{~d} \varphi_{i}
\end{aligned}
$$

where $\mathrm{d} \varphi_{i}$ is the observed variation of latitude referred to the fixed origin of the pole and $a_{i}, b_{i}, c_{i}$ are the constants which are given by the distribution of the stations and the following relations hold among them.

$$
\sum a_{i}=0, \quad \sum b_{i}=0, \quad \sum c_{i}=1 .
$$

In general the observed value of latitude variation $\mathrm{d} \varphi_{i}$ contains a local non-polar term (LNP) which is proper to each station. Therefore, we may assume that $\mathrm{d} \varphi_{i}$ is the sum of three terms-polar term, common $z$ term and local non-polar term.

Thus $\quad \mathrm{d} \varphi_{i}=\mathrm{d} \varphi_{i_{\mathrm{polar}}}+\mathrm{d} \varphi_{i_{\text {common } z}}+\mathrm{d} \varphi_{i_{\text {INP }}}$.

If $\mathrm{d} \varphi_{i_{\text {N P }}}=0$, we can get correct solutions from (1). However, if $\mathrm{d} \varphi_{i_{\text {INP }}} \neq 0$, then its disturbances $\Delta x, \Delta y$ and $\Delta z$ should be always contained in the least squares solutions.

That is, the solutions of the formula, the disturbing local non-polar terms

$$
\mathrm{d} \varphi_{i_{\mathrm{NP}}}=\Delta x \cos \lambda_{i}+\Delta y \sin \lambda_{i}+\Delta z
$$

are always contained in the solution of (1). 
The above relation is seen easily from Figure 1 in my previous paper (Okuda, 1968).

Figure 1 shows that if $\mathrm{d} \varphi_{i_{\mathrm{NP}}}=0$ (for example in the period 1955-1956), then we can get the same values of $x, y, z$ for 5 ILS stations, each combination of 4 ILS stations and even for 3 ILS (M.C.U.) stations. But, if $\mathrm{d} \varphi_{i_{\text {NP }}} \neq 0$, we can not get correct solutions for $x, y, z$.

This relation can be explained analytically as follows. Putting the solutions for 5 ILS stations and $4 \operatorname{ILS}(-i)$ stations as $x, y, z$ and $x^{\prime}, y^{\prime}, z^{\prime}$ respectively,

Then

$$
\begin{aligned}
& x=\sum a_{i} \mathrm{~d} \varphi_{i}, \quad y=\sum b_{i} \mathrm{~d} \varphi_{i}, \quad z=\sum c_{i} \mathrm{~d} \varphi_{i} \\
& x^{\prime}=\sum a_{i}^{\prime} \mathrm{d} \varphi_{i}, \quad y^{\prime}=\sum b_{i}^{\prime} \mathrm{d} \varphi_{i}, \quad z^{\prime}=\sum c_{i}^{\prime} \mathrm{d} \varphi_{i} .
\end{aligned}
$$

$$
\begin{aligned}
& \Delta x_{-i}=x-x^{\prime}=\sum\left(a_{i}-a_{i}^{\prime}\right) \mathrm{d} \varphi_{i} \\
& \Delta y_{-i}=y-y^{\prime}=\sum\left(b_{i}-b_{i}^{\prime}\right) \mathrm{d} \varphi_{i} \\
& \Delta z_{-i}=z-z^{\prime}=\sum\left(c_{i}-c_{i}^{\prime}\right) \mathrm{d} \varphi_{i} .
\end{aligned}
$$

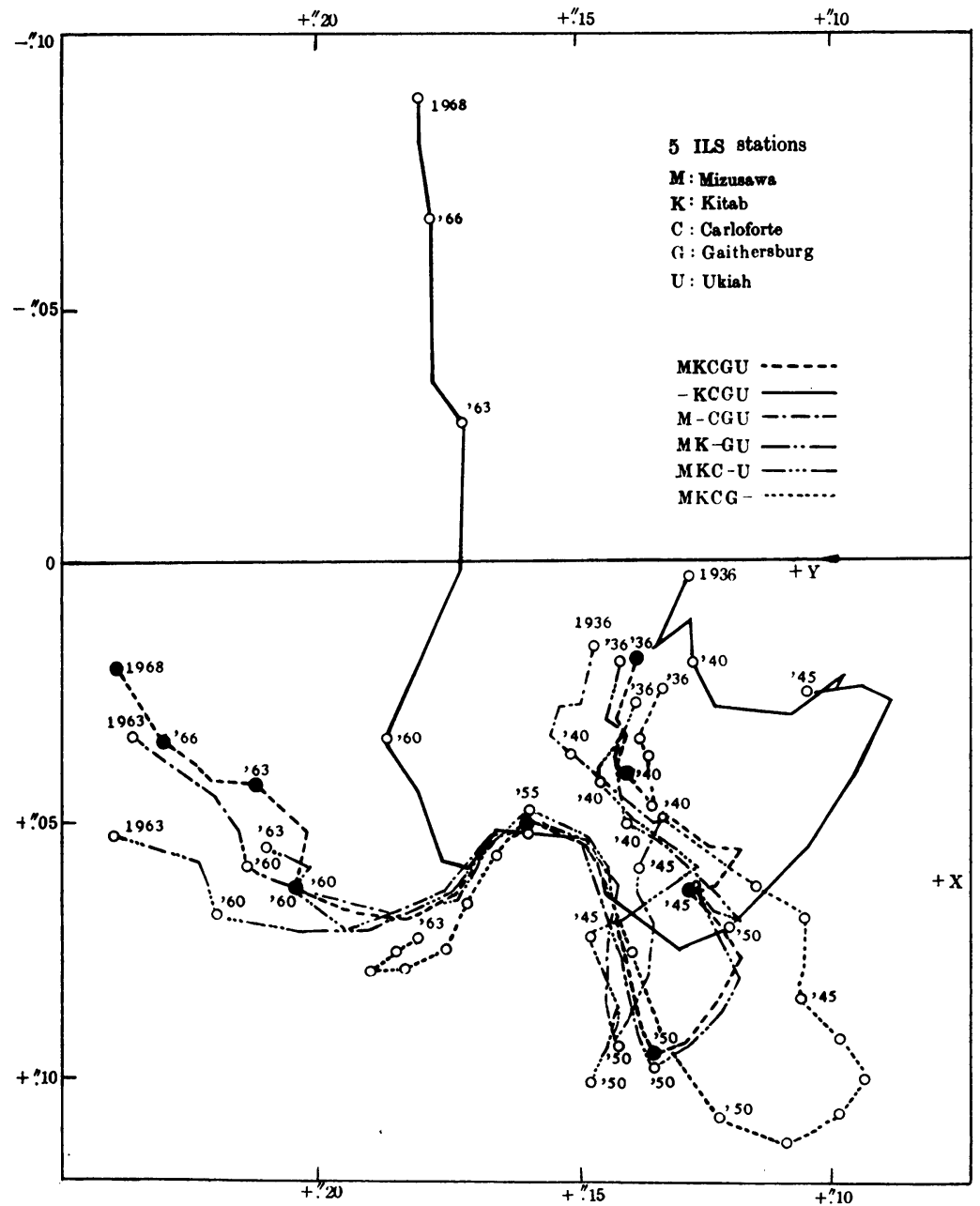

Fig. 1. 
From (3)

$$
\frac{\Delta x_{-i}}{\Delta z_{-i}}=\frac{\sum\left(a_{i}-a_{i}^{\prime}\right) \mathrm{d} \varphi_{i}}{\sum\left(c_{i}-c_{i}^{\prime}\right) \mathrm{d} \varphi_{i}}, \quad \frac{\Delta y_{-i}}{\Delta z_{-i}}=\frac{\sum\left(b_{i}-b_{i}^{\prime}\right) \mathrm{d} \varphi_{i}}{\sum\left(c_{i}-c_{i}^{\prime}\right) \mathrm{d} \varphi_{i}},
$$

whereas the following important relations hold among the coefficients as shown in Table I.

$$
\begin{aligned}
& \frac{a_{i}-a_{i}^{\prime}}{c_{i}-c_{i}^{\prime}}=\frac{a_{M}-a_{M}^{\prime}}{c_{M}-c_{M}^{\prime}}=\frac{a_{K}-a_{K}^{\prime}}{c_{K}-c_{K}^{\prime}}=\frac{a_{C}-a_{C}^{\prime}}{c_{C}-c_{C}^{\prime}}=\frac{a_{G}-a_{G}^{\prime}}{c_{G}-c_{G}^{\prime}}=\frac{a_{U}-a_{U}^{\prime}}{c_{U}-c_{U}^{\prime}}=K_{-i} \\
& \frac{b_{i}-b_{i}^{\prime}}{c_{i}-c_{i}^{\prime}}=\frac{b_{M}-b_{M}^{\prime}}{c_{M}-c_{M}^{\prime}}=\frac{b_{K}-b_{K}^{\prime}}{c_{K}-c_{K}^{\prime}}=\frac{b_{C}-b_{C}^{\prime}}{c_{C}-c_{C}^{\prime}}=\frac{b_{G}-b_{G}^{\prime}}{c_{G}-c_{G}^{\prime}}=\frac{b_{U}-b_{U}^{\prime}}{c_{U}-c_{U}^{\prime}}=K_{-i}^{\prime}
\end{aligned}
$$

where $K_{-i}$ and $K_{-i}$ are constant.

\section{TABLE I}

$i=M ; K_{-i}$ and $K_{-i}^{\prime}$

\begin{tabular}{llllll} 
& $a_{i}-a_{i}{ }^{\prime}$ & $b_{i}-b_{i}{ }^{\prime}$ & $C_{i}-C_{i}{ }^{\prime}$ & $K . M=\frac{a_{i}-a_{i}{ }^{\prime}}{C_{i}-C_{i}{ }^{\prime}} K^{\prime}-M=\frac{b_{i}-b_{i}{ }^{\prime}}{C_{i}-C_{i}{ }^{\prime}}$ \\
\hline$M$ & -0.4359 & -0.2636 & +0.2305 & -1.89 & -1.14 \\
$K$ & +0.4972 & +0.3007 & -0.2629 & -1.89 & -1.14 \\
$C$ & -0.2681 & -0.1622 & +0.1418 & -1.89 & -1.14 \\
$G$ & -0.2029 & -0.1227 & +0.1073 & -1.89 & -1.14 \\
$U$ & +0.4097 & +0.2478 & -0.2167 & -1.89 & -1.14 \\
\hline
\end{tabular}

And we get

$$
\begin{aligned}
& \frac{\Delta x_{-i}}{\Delta z_{-i}}=\frac{\sum\left(a_{i}-a_{i}^{\prime}\right) \mathrm{d} \varphi_{i}}{\sum\left(c_{i}-c_{i}^{\prime}\right) \mathrm{d} \varphi_{i}}=\frac{\left(a_{M}-a_{M}^{\prime}\right) \mathrm{d} \varphi_{M}}{\left(c_{M}-c_{M}^{\prime}\right) \mathrm{d} \varphi_{M}}= \\
& =\frac{\left(a_{K}-a_{K}^{\prime}\right) \mathrm{d} \varphi_{K}}{\left(c_{K}-c_{K}^{\prime}\right) \mathrm{d} \varphi_{K}}=\frac{\text { so on for C.G.U. }}{\text { so on for C.G.U. }}=K_{-i} \\
& \frac{\Delta y_{-i}}{\Delta z_{-i}}=\frac{\sum\left(b_{i}-b_{i}^{\prime}\right) \mathrm{d} \varphi_{i}}{\sum\left(c_{i}-c_{i}^{\prime}\right) \mathrm{d} \varphi_{i}}=\frac{\left(b_{M}-b_{M}^{\prime}\right) \mathrm{d} \varphi_{M}}{\left(c_{M}-c_{M}^{\prime}\right) \mathrm{d} \varphi_{M}}= \\
& =\frac{\left(b_{K}-b_{K}^{\prime}\right) \mathrm{d} \varphi_{K}}{\left(c_{K}-c_{K}^{\prime}\right) \mathrm{d} \varphi_{K}}=\frac{\text { so on for C.G.U. }}{\text { so on for C.G.U. }}=K_{-i}^{\prime} \\
& \Delta x_{-i}=K_{-i} \Delta z_{-i} \\
& \Delta y_{-i}=K_{-i}^{\prime} \Delta z_{-i} \quad(i \geqq 4)
\end{aligned}
$$

The relation (4) can hold without any restriction in number of station.

In the case of 5 ILS and 4 ILS $(-M)$, by using (4) the disturbing local non-polar term $\mathrm{d} \varphi_{I \mathrm{NP}}$ which correspond to $\Delta x_{-M}, \Delta y_{-M}$, is

$$
\begin{aligned}
\mathrm{d} \varphi_{M_{I N P}} & =\left(\Delta x_{-M} \cos \lambda_{M}+\Delta y_{-M} \sin \lambda_{M}\right) \\
& =\Delta z_{-M}\left(K_{-M} \cos \lambda_{M}+K_{-M}^{\prime} \sin \lambda_{M}\right) .
\end{aligned}
$$




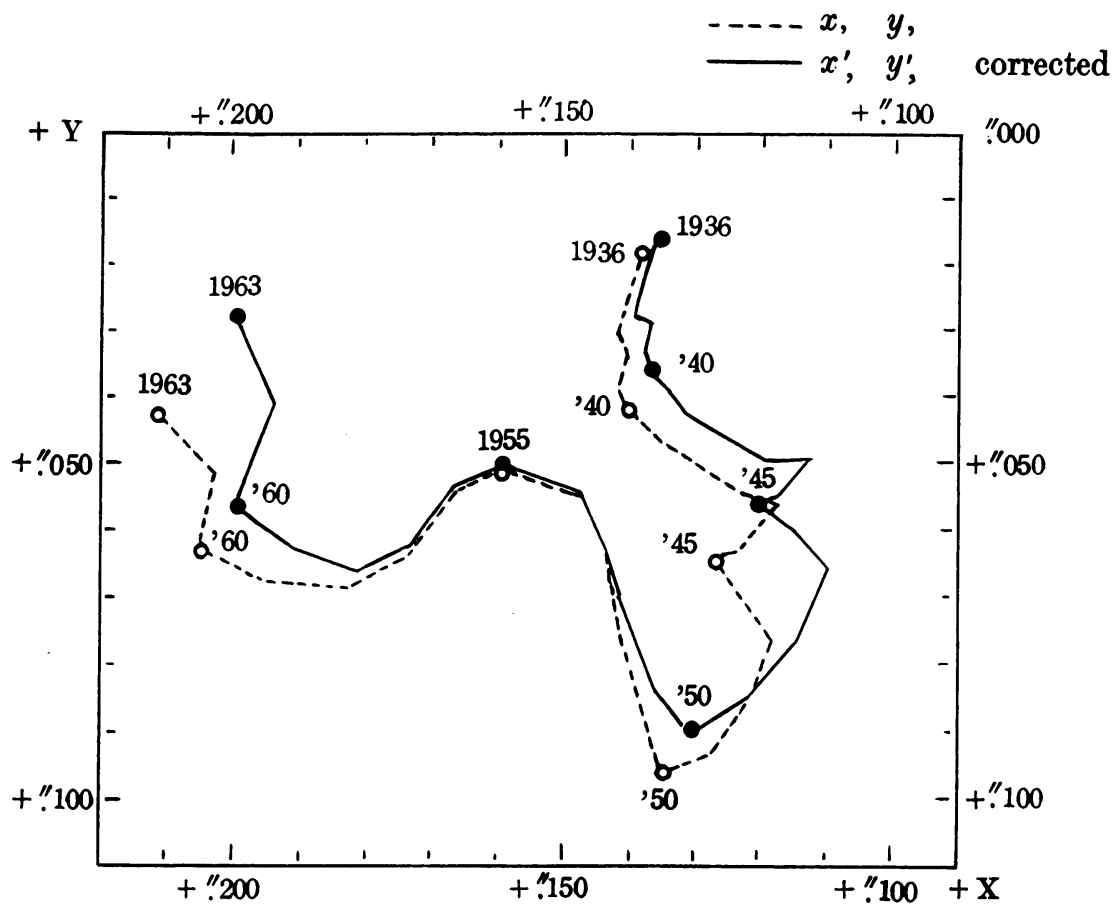

Fig. 2. Motion of the mean pole.

As the same relation can hold for other ILS stations, we may compute $d \varphi_{K_{\text {INP }} \text {, }}$ $\mathrm{d} \varphi_{C_{I N \mathrm{NP}}}, \mathrm{d} \varphi_{G_{I N P}}, \mathrm{~d} \varphi_{U_{I N P}}$ and the disturbances $\Delta x, \Delta y, \Delta z$ for the least squares solutions of 5 ILS stations as follows.

$$
\begin{aligned}
\Delta x & =\sum a_{i} \mathrm{~d} \varphi_{i_{\mathrm{NP}}} \\
\Delta y & =\sum b_{i} \mathrm{~d} \varphi_{i_{\mathrm{NP}}} \\
\Delta z & =\sum c_{i} \mathrm{~d} \varphi_{i_{\text {INP }}}
\end{aligned} \quad i=M, K, C, G, U .
$$

Applying these corrections, the corrected polar coordinates of the mean pole are computed and the result is given in Figure 2.

The local non-polar term described above is only the part which corresponds to disturbance upon the polar coordinates and common $z$ term independent of the polar motion. The total amount of the local non-polar term for each station is deduced.

where

$$
\mathrm{d} \varphi_{i_{\mathrm{INP}}}=\mathrm{d} \varphi_{i}-\left\{x_{\text {corr }} \cos \lambda_{i}+y_{\text {corr }} \sin \lambda_{i}+z_{\text {corr }}\right\},
$$

$$
\begin{aligned}
& x_{\text {corr }}=x-\Delta x=\sum a_{i} \mathrm{~d} \varphi_{i}-\Delta x \\
& y_{\text {corr }}=y-\Delta y=\sum b_{i} \mathrm{~d} \varphi_{i}-\Delta y \\
& z_{\text {corr }}=z-\Delta z=\sum c_{i} \mathrm{~d} \varphi_{i}-\Delta z .
\end{aligned}
$$

The total local non-polar terms computed by formula (7) are just the same as $\sum\left(z_{5}-z_{4}\right)$ 
in the previous paper (Okuda, 1968) and this coincidence proves the correctness of the results obtained by author. The conclusion in this section may be summarized as follows.

When the amount of $(o-c)$ is beyond the range of observation errors (p.e. $\pm 0.2 \sim 0$ ". 1 for a single pair), the polar coordinates computed by usual computation formula (1) are considered to be revised by the method described above.

\section{In the Case of Time Observation}

We use the following formula for computing the polar coordinates $x, y$ and the common rotation term $T$ independent of the polar motion.

$$
\mathrm{d} \tau_{i}=\left(-x \sin \lambda_{i}+y \cos \lambda_{i}\right) \tan \varphi_{i}+T \quad(i \geqq 3)
$$

and the solutions of these formulae by the method of least squares are

$$
\begin{aligned}
x & =\sum A_{i} \mathrm{~d} \tau, \\
y & =\sum B_{i} \mathrm{~d} \tau, \\
T & =\sum C_{i} \mathrm{~d} \tau,
\end{aligned}
$$

where $\mathrm{d} \tau_{i}$ is the observed variation of time referred to the fixed origin of the pole and $A_{i}, B_{i}, C_{i}$ are the constants and the following relations hold among them in just the same way as in the case of latitude variation.

$$
\sum A_{i}=0, \quad \sum B_{i}=0, \quad \sum C_{i}=1 .
$$

From the analogy in the case of latitude variation, we can derive the local non-polar term $\mathrm{d} \tau_{i_{\mathrm{NP}}}$ which disturbs the least squares solutions of (2.1) as below.

If $\mathrm{d} \tau_{i_{\mathrm{NP}}} \neq 0$, then the disturbances $\Delta x, \Delta y$ and $\Delta T$ are always contained in the least squares solutions unless the distribution of the stations and the local non-polar terms satisfy the conditions

$$
\sum \sin \lambda_{i} \tan \varphi_{i}=0, \quad \sum \cos \lambda_{i} \tan \varphi_{i}=0, \quad \sum \mathrm{d} \tau_{i_{i \mathrm{NP}}}=0
$$

or

$$
\sum \sin \lambda_{i} \cot \varphi_{i}=0, \quad \sum \cos \lambda_{i} \cot \varphi_{i}=0, \quad \sum \mathrm{d} \tau_{i_{\text {NP }}}=0 .
$$

That is, the solutions for the formula

$$
\mathrm{d} \tau_{i_{\mathrm{NP}}}=-\Delta x \sin \hat{\lambda}_{i}+\Delta y \cos \lambda_{i}+\Delta T
$$

are always contained in the solutions of (2.1).

We can compute the local non-polar term $\mathrm{d} \tau_{i_{\text {INP }}}$ for the $i$-th station by utilizing the difference between solutions for $n$ stations and $n-1$ stations $(-i)$ through the same procedure described in $\S 1$.

$$
\begin{aligned}
\mathrm{d} \tau_{i_{I N P}} & =\Delta T_{-i}\left(-L_{-i} \sin \lambda_{i}+L_{-i}^{\prime} \cos \lambda_{i}\right) \tan \varphi_{i} \\
& =\Delta T_{-i}\left(-L_{-i} \sin \lambda_{i}+L_{-i}^{\prime} \cos \lambda_{i}\right) \tan \varphi_{i}
\end{aligned}
$$

where $L_{-i}$ and $L_{-i}^{\prime}$ are constants. 
And

$$
\begin{aligned}
\Delta x & =\sum A_{i} \mathrm{~d} \tau_{i_{\mathrm{NP}}} \\
\Delta y & =\sum B_{i} \mathrm{~d} \tau_{i_{\mathrm{NP}}} \quad i=1,2 \ldots n \\
\Delta T & =\sum C_{i} \mathrm{~d} \tau_{i_{\text {INP }}}
\end{aligned}
$$

The total amount of the local non-polar term for each station are deduced

where

$$
\mathrm{d} \tau_{i_{\mathrm{INP}}}=\mathrm{d} \tau_{i}-\left\{\left(-x_{\mathrm{corr}} \sin \lambda_{i}+y_{\text {corr }} \cos \dot{\lambda}_{i}\right) \tan \varphi_{i}+T_{\mathrm{corr}}\right\}
$$

$$
\begin{aligned}
& x_{\text {corr }}=x-\Delta x=\sum A_{i} \mathrm{~d} \tau_{i}-\Delta x \\
& y_{\text {corr }}=y-\Delta y=\sum B_{i} \mathrm{~d} \tau_{i}-\Delta y \\
& T_{\text {corr }}=T-\Delta T=\sum C_{i} \mathrm{~d} \tau_{i}-\Delta T .
\end{aligned}
$$

Formulae (6) and (2.6) show that the local non-polar term always disturbs the least squares solutions and $\Delta x, \Delta y$ in the computed polar coordinates are proportional to $\Delta z, \Delta T$ respectively, and the constants of its ratio are determined by the coefficients of the conditional equations.

\section{Some Test Example}

A comparison between Okazaki's (Okazaki and Nasaka, 1971) $T$ terms which were deduced as the least square's solutions of $d \tau_{i}$ of 11 stations (PZT and Astrolabe) for the period 1964-1966 and Iijima and Okazaki's annual and semi-annual rotation terms $\left(T, T^{1 / 2}\right)$ which were deduced from UT2-A3 (BIH) for the period 1955-1968 by spectrum analysis has been made. And the approximate estimation of the fictitious rotation term $\Delta T$ in Okazaki's $T$ term is derived for the mean year of 1964, 1965, 1966 as below.

$$
\begin{aligned}
\Delta T & \fallingdotseq T_{\text {obs }}(\text { Okazaki })-\left(T+T^{1 / 2}\right)-(a+b t) \\
& =5^{\mathrm{ms}} \cdot 1 \cos \left(2 \pi t+183^{\circ}\right) .
\end{aligned}
$$

On the other hand, the differences between annual terms obtained by using time and latitude observations by Okazaki are, neglecting constant and linear term of $t$,

(Time)-(Latitude)

$$
\begin{aligned}
& \Delta x=x_{\tau}-x_{\varphi} \fallingdotseq-0.04 \cos 2 \pi t, \text { for the period 1955-68 } \\
& \Delta y=y_{\tau}-y_{\varphi} \fallingdotseq+0.022 \cos 2 \pi t, \text { mean of } 1964,65,66,
\end{aligned}
$$

where $t$ is a fraction of year counted from the beginning of year.

These values correspond to

$$
\Delta x \fallingdotseq 0.52 \Delta T, \quad \Delta y=-0.27 \Delta T \text {. }
$$

In the case of Guinot and Feissel (1968).

Utilizing their annual terms obtained by the observation data for $18 \mathrm{PZT}$ and Astrolabe, the following differences are derived. 
(Time)-(Latitude)

$$
\begin{array}{ll}
\Delta x=x_{\tau}-x_{\varphi}=-0^{\prime \prime} 06 \cos \left(2 \pi t+50^{\circ}\right), & \text { for the period } \\
\Delta y=y_{\tau}-y_{\varphi}=+0^{\prime \prime} 05 \cos \left(2 \pi t+50^{\circ}\right), & \text { mean of } 1964,65,66
\end{array}
$$

Time $(G$ and $F)-($ ILS)

$$
\begin{aligned}
& \Delta x=x_{\tau}-x_{\mathrm{ILS}} \fallingdotseq-0^{\prime \prime} 035 \cos \left(2 \pi t+68^{\circ}\right) \\
& \Delta y=y_{\tau}-y_{\mathrm{ILS}} \fallingdotseq+0^{\prime \prime} 047 \cos \left(2 \pi t+68^{\circ}\right) .
\end{aligned}
$$

We may assume that $\Delta T=6^{\mathrm{ms}} \sim 5^{\mathrm{ms}} \cos \left(2 \pi t+230^{\circ}\right)$ would be contained in their $T$ term for the mean year 1965 .

\section{Conclusion}

The interpretation of the ambiguity between annual terms obtained by time and latitude observations is a difficult and complicated problem. The study, however, on this ambiguity is very important for our purpose to obtain more reliable polar coordinate, common terms $z$ and $T$.

Utilizing the method described in this paper, we may compute a revised coordinates which are comparable to each other in both annual terms obtained by time and latitude observation. And at the same time we are able to study the characters of the nonpolar variations in time or latitude observation which is proper to each station by subtracting correct amount of polar effect.

According to the variation of $\Delta T$ with time, the changes in the phase angle for the annual term and the amount of $\Delta x, \Delta y$ which are differences between both annual terms obtained by time and latitude observations will be caused.

\section{References}

Guinot, B. and Feissel, M.: 1968, in Wm. Markowitz and B. Guinot (eds.), 'Continental Drift, Secular Motion of the Pole, and Rotation of the Earth', IAU Symp. 32, 63.

Okazaki, S. and Nasaka, M.: 1971, Ann. Tokyo Astron. Obs. 12, No. 3.

Okuda, T.: 1968, Publ. Int. Lat. Obs. Mizusawa 6, No. 2. 